\title{
Perianal Pain as a Presentation of Lumbosacral Neurofibroma: A Case Report
}

\author{
Mehdi Moghaddasi $^{1} \quad$ Mahboubeh Aghaii $^{1} \quad$ Mansoureh Mamarabadi $^{1}$ \\ ${ }^{1}$ Department of Neurology, Rasool Akram Hospital, Tehran University \\ of Medical Sciences, Tehran, Iran \\ J Neurol Surg Rep 2014;75:e191-e193. \\ Address for correspondence Mehdi Moghaddasi, Associate Professor \\ of Neurology, Department of Neurology, Tehran University of Medical \\ Sciences, Tehran, Iran; Postal code: 1445613131 \\ (e-mail: moghaddasim@hotmail.com).
}

\begin{abstract}
Keywords

- perianal pain

- referral pain

- neurofibroma

- proctalgia

Rectal and perianal pain is a common problem. Most people have experienced it at least once in their lifetime. It usually manifests as mild discomfort, but sometimes the pain can be so severe that it is incapacitating. A 59-year-old woman admitted with a 2-year history of paroxysmal perianal pain underwent a full work-up including proctoscopy, sigmoidoscopy, full colonoscopy, and barium enema that were unremarkable. Lumbosacral magnetic resonance imaging with and without gadolinium showed an intraduralextramedullary lesion at the level of $\mathrm{L} 5$. The pathologic diagnosis was a neurofibroma. She underwent surgery, and after a few weeks she felt well and medication was no longer needed for her paroxysmal pain. Although one should consider the usual causes of colorectal pain such as hemorrhoids, anal fissure, proctalgia fugax, and chronic perianal pain syndrome, we should keep in mind that some referral pain may mimic local pathologies and should be evaluated properly.
\end{abstract}

\section{Introduction}

Rectal and perianal pain is common. ${ }^{1}$ It is usually perceived as mild discomfort, but sometimes it is incapacitating. It may be due to hemorrhoids, anal fissure, proctalgia fugax, or chronic perianal pain syndrome, and less commonly to cancers, proctitis, rectal prolapse, and inflammatory bowel diseases. ${ }^{2}$ The diagnosis is based on history, physical examination, and ancillary work-up. The pain of proctalgia fugax is sudden and intense, usually lasting less than a minute. But in rare cases, the spasms can continue for an hour. The pain is described as sharp, stabbing, or cramp-like occurring at the anal opening. It may awaken the person during sleep. The attacks occur in clusters, appearing daily for a while and then disappearing for weeks or months. ${ }^{3}$ Pain related to the levator ani syndrome is a constant or frequently occurring dull pain that is felt higher up inside the rectal passage. Ancillary tests are performed according to the history and examination, and they may consist of a rectal examination, proctoscopy or full colonoscopy, and in some instances a barium enema.

received

October 26, 2012

accepted

September 1, 2013

published online

July 21, 2014

\section{Case Report}

A 59-year-old woman was admitted to Rasool-Akram Hospital (affiliated with the Tehran University of Medical Sciences, Tehran, Iran) with a 2-year history of paroxysmal perianal pain. Her physical examination including both rectal and pelvic examinations were normal. She underwent a full work-up including proctoscopy, sigmoidoscopy, full colonoscopy, and barium enema, all of which were unremarkable. Then she was prescribed some anxiolytics and gabapentin with the diagnosis of neuralgic pain and referred to the neurology clinic. Lumbosacral magnetic resonance imaging (MRI) was performed for an evaluation of the referred pain (-Fig. 1). The lumbosacral MRI showed an extramedullary-intradural lesion at the level of L5 with fairly uniform enhancement. She underwent surgery and after few weeks felt well. Her paroxysmal pain was gone, and she no longer needed medication for the pain (-Fig. 2). The pathologic diagnosis was neurofibroma.
License terms

Stuttgart · New York

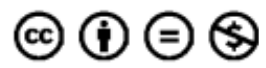

DOI http://dx.doi.org/

10.1055/s-0033-1358793. ISSN 2193-6358. 


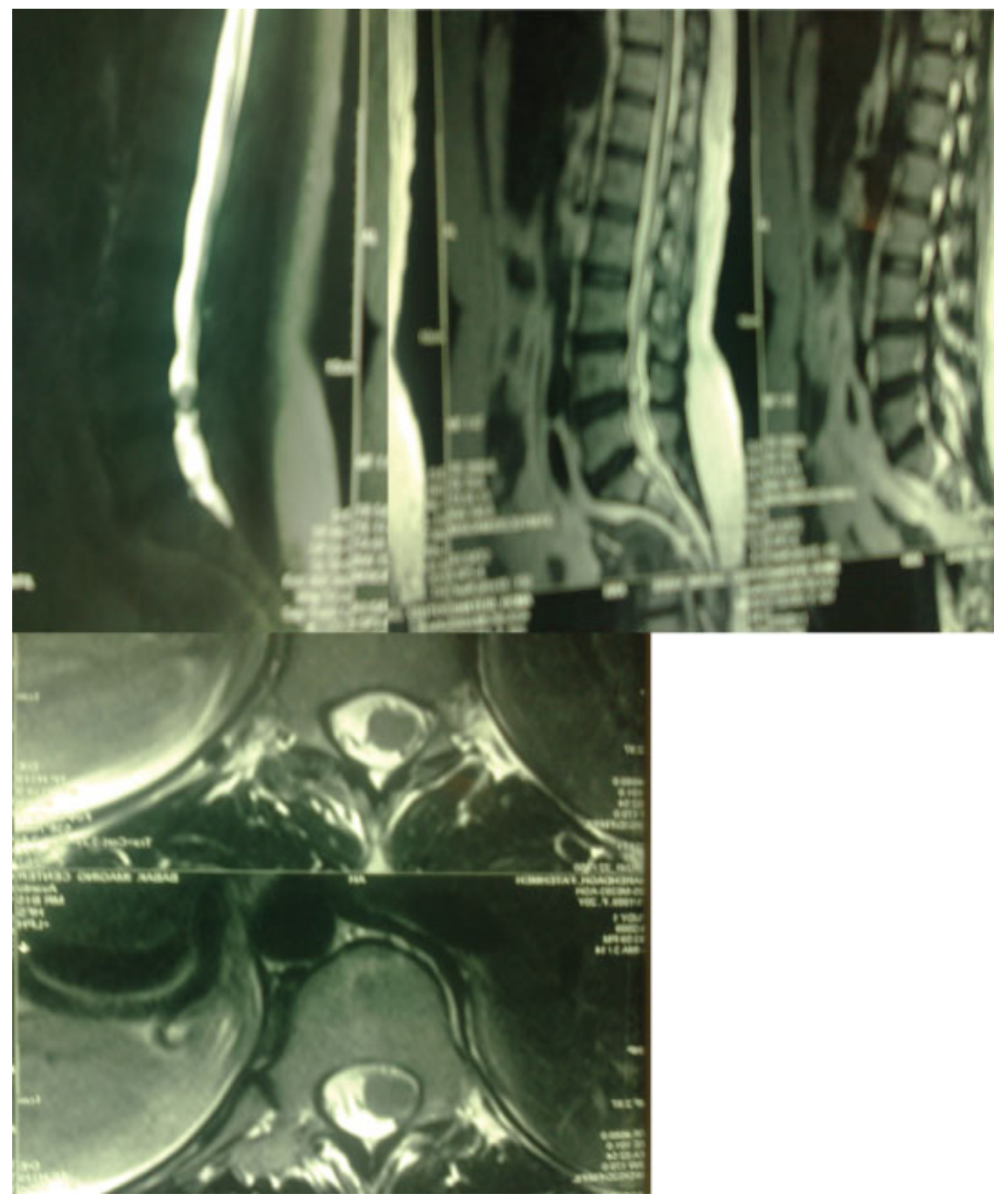

Fig. 1 Myelogram, sagittal, and axial T2 of lumbosacral magnetic resonance imaging show an extramedullary lesion.

\section{Conclusions}

Although one should consider the usual causes of colorectal pain such as hemorrhoids, anal fissure, anal herpes, ulcerative proctitis, perianal hematoma, perianal or ischiorectal abscess, pilonidal sinus, perianal warts, anal carcinoma, proctalgia fugax, and chronic perianal pain syndrome, ${ }^{4}$ we should keep in mind that some referred pain may mimic local pathologies and should be evaluated properly. Nerve sheath tumors are either benign or malignant. The benign variants are schwannoma (neurilemoma) and neurofibroma. Neurofibromas are fibroblastic neoplasms of peripheral nerves that are benign tumors. ${ }^{5}$ They account for $~ 16$ to $30 \%$ of all spinal tumors and $13.7 \%$ of spinal tumors of nerve sheath origin. ${ }^{6}$ Neurofibromas and schwannomas are the most common spinal tumors that are evenly distributed along the spinal canal. The thoracic spine is the most common site for neurofibroma. Cervical and lumbosacral regions are other sites of this tumor. About 1 to $55 \%$ of spinal neurofibromas are located in the sacral region. ${ }^{7,8}$ Multiple and plexiform neurofibromas are associated with von Recklinghausen disease (neurofibromatosis type 1 [NF1]). Neurofibromas are rarely solitary and nonen- capsulated. Cutaneous solitary neurofibroma may be a typical and exclusive lesion of NF1, although $\sim 90 \%$ of central and peripheral neurofibromas may be solitary and unrelated to systemic NF. ${ }^{5,9,10}$ The imaging characteristics of neurofibromas depend on their fibrous and myxoid tissues. Of nerve sheath origin, they occur as a fusiform enlargement of the nerve, and neural fibers are dispersed within the lesion. Neurofibromas are slow growing and noninvasive, and their elastic consistency permits them to be shaped by adjacent bone structures; the bone reacts by remodeling around the lesions. They rarely calcify and frequently have a bibbed "dumbbell" appearance. On MRI, neurofibromas are isointense with muscle on T1-weighted images and show a hypersignal on T2-weighted images. They may be heterogeneous. ${ }^{11}$ The most common signs and symptoms are due to compression of the affected nerve root. The primary treatment for neurofibromas is surgical resection. The neurologic outcome is related to the tumor bulk and whether or not the adjacent nerve root can be spared. ${ }^{12}$ Malignant degeneration to neurofibrosarcoma occurs in 4 to $11 \%$ of patients with neurofibromatosis. ${ }^{13}$ Teichmann et al reported a case of solitary 

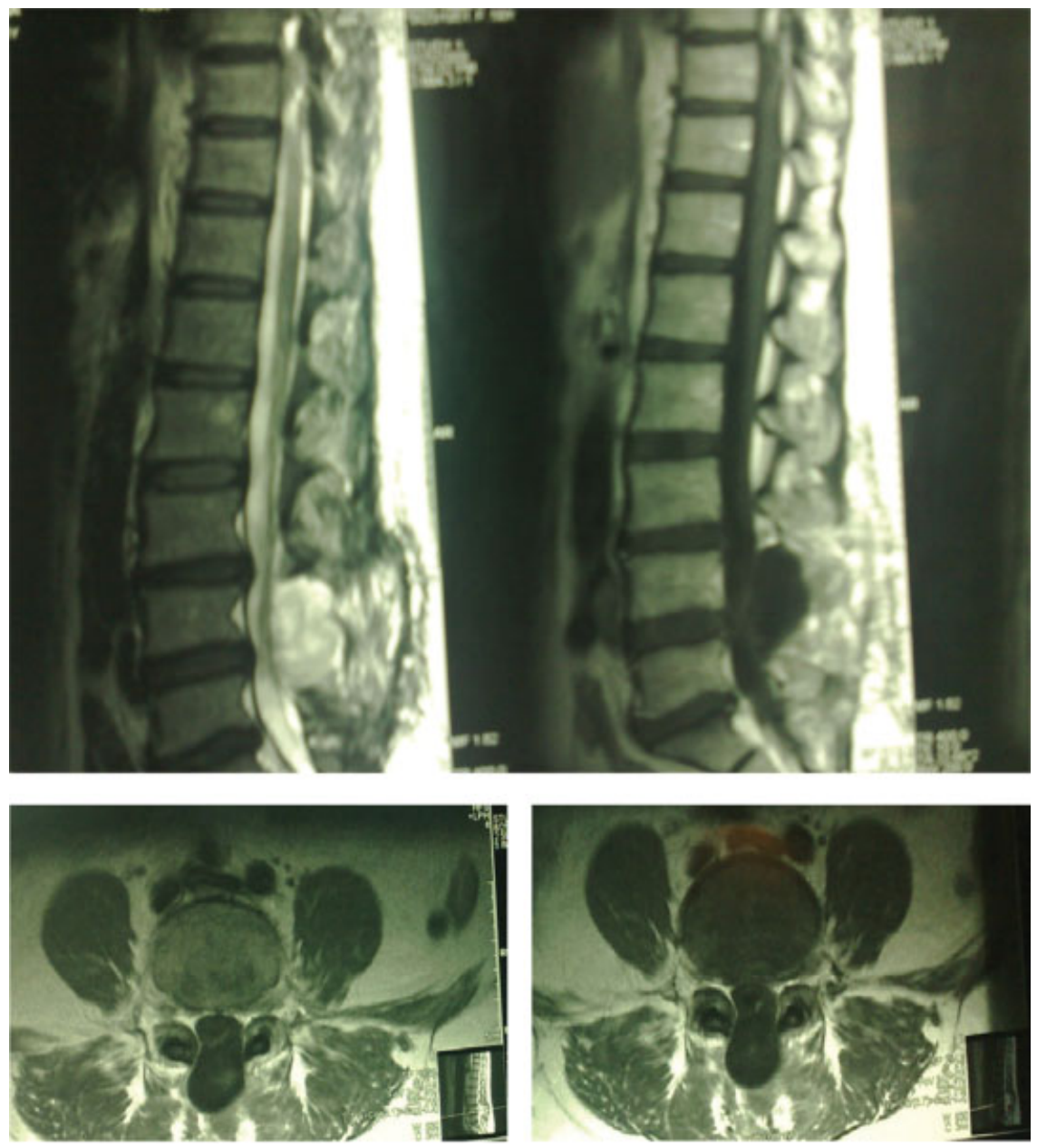

Fig. 2 Postoperative lumbosacral magnetic resonance imaging shows removal of the lesion with meningocele formation.

neurofibroma of the lumbosacral plexus causing pain and leg paresis. ${ }^{14}$ Daniel and his colleagues reported a young woman with low back pain and a left $\mathrm{S} 1$ nerve root mass enlarging the neural foramen and extending extradurally into the presacral soft tissues with a final diagnosis of neurofibroma. ${ }^{15}$

\section{Disclaimer}

The authors have no conflicts of interest to disclose.

\section{References}

1 Wesselmann U, Burnett AL, Heinberg LJ. The urogenital and rectal pain syndromes. Pain 1997;73(3):269-294

2 Kornel EE, Vlahakos D. Intraspinal schwannoma presenting solely with rectal pain. Neurosurgery 1988;22(2):417-419

3 Haraguchi M, Kinoshita H, Koori M, et al. Multiple rectal carcinoids with diffuse ganglioneuromatosis. World J Surg Oncol 2007;5:19

4 Daniel WJ. Anorectal pain, bleeding and lumps. Aust Fam Physician 2010;39(6):376-381

5 Harkin JC, Reed RJ. Tumors of the Peripheral Nervous System. Washington, DC: Armed Forces Institute of Pathology; 1969: 51-97
6 Seppälä MT, Haltia MJJ, Sankila RJ, Jääskeläinen JE, Heiskanen O. Long-term outcome after removal of spinal neurofibroma. J Neurosurg 1995;82(4):572-577

7 Benerji AK. Epidemiology of CNS tumors 1974-1978. In: Proceedings of the National Seminar on Neurooncology. Bangalore, India: National Institute of Mental Health and Neurosciences; 1981: 111-143

8 Rao SB. Spinal neurinoma. A study of 80 operated cases. Neurol India 1975;23(1):1-12

9 Das Gupta TK, Brasfield RD, Strong EW, Hajdu SI. Benign solitary schwannomas (neurilemomas). Cancer 1969;24(2):355-366

10 Sanguinetti C, Specchia N, Gigante A, de Palma L, Greco F. Clinical and pathological aspects of solitary spinal neurofibroma. J Bone Joint Surg Br 1993;75(1):141-147

11 Breidahl WH, Khangure MS. MRI of lumbar and sacral plexus nerve sheath tumours. Australas Radiol 1991;35(2):140-144

12 Feldenzer JA, McGauley JL, McGillicuddy JE. Sacral and presacral tumors: problems in diagnosis and management. Neurosurgery 1989;25(6):884-891

13 Zimmerman RA, Bilaniuk LT. Imaging of tumors of the spinal canal and cord. Radiol Clin North Am 1988;26(5):965-1007

14 Argyrakis A, Teichmann A, Kuhn W. Solitary neurofibroma of the lumbosacral plexus. J Neurol Neurosurg Psychiatry 1985;48(8): 844-846

15 Barboriak DP, Rivitz SM, Chew FS. Sacral neurofibroma. AJR Am J Roentgenol 1992;159(3):600 